Bull. Austral. Math. Soc.

VOL. 57 (1998) [363-365]

\title{
CENTRALISERS OF GALOIS GROUP ACTIONS
}

\author{
MARK KISIN
}

If $f$ is a polynomial over a field $E$, and $\sigma$ a permutation of the roots of $f$, we show that $\sigma$ is given by a polynomial $p$ with coefficients in $E$ (that is, $p(a)=\sigma(a)$ for each root $a$ of $f$ ) if and only if $\sigma$ commutes with all the Galois automorphisms.

If $E$ is a field, and $\bar{E}$ a separable closure of $E$, then $\mathrm{Gal}(\bar{E} / E)$ acts on the roots of unity in $\bar{E}$ via the cyclotomic character. In particular the action of each Galois automorphism on roots of unity is a polynomial function. The purpose of this note is to point out that this is quite a general phenomenum. Namely we give two proofs of the following

THEOREM. Let $E$ be a field, $f \in E[X]$, a polynomial with distinct roots, and $\sigma$ a permutation of the roots of $f$. There is a polynomial $p \in E[X]$ such that $p(a)=\sigma(a)$ for each root $a$ of $f$ if and only if $\sigma$ commutes with the Galois action on the roots of $f$.

In particular, if the roots of $f$ generate an Abelian extension of $E$ then the Galois action on the roots is given by polynomials in the sense above.

The theorem is pretty but not deep, and both proofs are completely elementary.

First Proof: We denote by $G$ the absolute Galois group of $E$. The "only if" part is clear, since then for each $s \in G$ and root $a$ of $f$ we must have

$$
s(\sigma(a))=s(p(a))=p(s(a))=\sigma(s(a)) .
$$

Now suppose that $\sigma$ commutes with the Galois action on the roots. If $f=f_{1} f_{2}$. factors over $E$, then $f_{1}$ and $f_{2}$ are coprime. If $p_{1}, p_{2} \in E[X]$ are such that $p_{1}(a)=$ $\sigma(a), p_{2}(b)=\sigma(b)$ for each root $a$ of $f_{1}$ and $b$ of $f_{2}$ then we may choose $p$ congruent to $p_{1}$ modulo $f_{1}$ and $p_{2}$ modulo $f_{2}$.

Thus we may assume $f$ is irreducible. Let $a$ be a root of $f$, and $s \in G$. If $s(a)=a$ then $s(\sigma(a))=\sigma(s(a))=\sigma(a)$. So $s$ fixes $\sigma(a)$ and $\sigma(a) \in E[a]$. Thus $\sigma(a)=p(a)$ for some $p \in E[X]$. If $b$ is another root of $f$, then $b=t(a)$ for some $t \in G$, since $f$ is

Received 20th August, 1997

Copyright Clearance Centre, Inc. Serial-fee code: 0004-9729/98 \$A2.00+0.00. 
irreducible. Hence $\sigma(b)=\sigma(t(a))=t(\sigma(a))=t(p(a))=p(t(a))=p(b)$, and $p$ is the required polynomial.

SECOND PROOF: We retain the notation above, and give a second proof of the "if" part.

Let $a_{1}, \ldots, a_{n}$ be the roots of $f$, and denote by $f_{i}(1 \leqslant i \leqslant n)$ the polynomial $f(X) /\left(X-a_{i}\right)$. We set

$$
p(X)=\sum_{i=1}^{n}\left[f_{i}(X) / f_{i}\left(a_{i}\right)\right] \sigma\left(a_{i}\right)
$$

This is the Lagrange interpolation formula, and we have $p\left(a_{i}\right)=\sigma\left(a_{i}\right)$ for each $i$.

To see that $p \in E[X]$ we extend the action of $G$ to $E\left[a_{1}, \ldots, a_{n}\right][X]$ by letting $G$ act on the coefficients of polynomials. If $s \in G$, choose $w \in S_{n}$ such that $s\left(a_{i}\right)=a_{w(i)}$. We get

$$
\begin{aligned}
s(p(X)) & =\sum_{i=1}^{n}\left[f_{w(i)}(X) / f_{w(i)}\left(a_{w(i)}\right)\right] s\left(\sigma\left(a_{i}\right)\right)=\sum_{i=1}^{n}\left[f_{w(i)}(X) / f_{w(i)}\left(a_{w(i)}\right)\right] \sigma\left(s\left(a_{i}\right)\right) \\
& =\sum_{i=1}^{n}\left[f_{w(i)}(X) / f_{w(i)}\left(a_{w(i)}\right)\right] \sigma\left(a_{w(i)}\right)=p(X) .
\end{aligned}
$$

An amusing application of the above theorem is a proof of the following theorem on permutation groups [1, Theorem $4.2 \mathrm{~A}]$.

CoRollary. Let $H \subset S_{n}$ be a transitive permutation group. Then the only element of the centraliser of $H$ in $S_{n}$ which has a fixed point is the identity.

In particular if $H$ is Abelian it is its own centraliser.

Proof: Let $E$ and $f$ be as in the theorem. Denote by $J$ the group of permutations of the roots of $f$ induced by $G$. Choose $E$ and $f$ so that $J$ is isomorphic to $H$ as a permutation group. This is possible, since one can choose $E$ and $f$ so that $J \stackrel{\sim}{\longrightarrow} S_{n}$ and then replace $E$ by a finite extension.

If $\sigma \in S_{n}$ commutes with all the elements of $J$ then by the theorem we have $\sigma(a)=p(a)$ for some $p \in E[X]$ and each root $a$ of $f$. If $\sigma(a)=a$ then we have $p(a)=a$. Since $p$ can be chosen so that $\operatorname{deg} p<\operatorname{deg} f=n$, and $f$ is irreducible, we see that $p(X)=X$ and $\sigma=$ id.

Finally we pose the following question:

Suppose that $E$ is a local field, and that we are given a sequence $f_{1}, f_{2}, \ldots$ of polynomials in $E[X]$ with distinct roots and such that $f_{i} \mid f_{i+1}$ for each $i$. We let $\mathcal{R}$ denote the union of the roots of the $f_{i}$. If $\sigma$ is an automorphism of $\mathcal{R}$, when is there a power series $p$ with coefficients in $E$ such that $p(a)=\sigma(a)$ for each $a \in \mathcal{R}$ ? In 
particular the equality means that the left hand side is well defined. Clearly $\sigma$ must commute with the Galois action on $\mathcal{R}$, but this is perhaps not sufficient because of convergence problems.

We remark that if $\mathcal{R}$ is the set of torsion points of a Lubin - Tate formal group over the ring of integers of $E$, (so the Galois action on $\mathcal{R}$ is Abelian) and $\sigma$ is induced by a Galois automorphism, then the required $p$ exists [2, Proposition 4.9]. The situation where $\mathcal{R}$ consists of elements of the form $\zeta-1$ with $\zeta$ a power root of unity is a special case, the power series associated to $\sigma \in G^{\mathrm{ab}}$ being $p(X)=(1+X)^{\omega(\sigma)}-1$, where $\omega: G^{\mathrm{ab}} \longrightarrow \mathbb{Z}_{p}^{*}$ is the cyclotomic character.

\section{REFERENCES}

[1] J. Dixon and B. Mortimer, Permutation groups, Graduate Texts in Mathematics 163 (Springer-Verlag, Berlin, Heidelberg, New York, 1996).

[2] K. Iwasawa, Local class field theory, Oxford Mathematical Monographs (Oxford University Press, New York, 1986).

Department ofMathematics

University of Sydney

Sydney NSW 2006

Australia 